MALYTSKA G.P., BURTNYAK I.V.

\title{
CONSTRUCTION OF THE FUNDAMENTAL SOLUTION OF A CLASS OF DEGENERATE PARABOLIC EQUATIONS OF HIGH ORDER
}

\begin{abstract}
In the article, using the modified Levy method, a Green's function for a class of ultraparabolic equations of high order with an arbitrary number of parabolic degeneration groups is constructed. The modified Levy method is developed for high-order Kolmogorov equations with coefficients depending on all variables, while the frozen point, which is a parametrix, is chosen so that an exponential estimate of the fundamental solution and its derivatives is conveniently used.

Key words and phrases: degenerated parabolic equations, modified Levy's method, Kolmogorov's equation, fundamental solution, parametrix.
\end{abstract}

Vasyl Stefanyk Precarpathian National University, 57 Shevchenka str., 76018, Ivano-Frankivsk, Ukraine E-mail: ivan.burtnyakepnu.edu.ua (Burtnyak I.V.)

\section{INTRODUCTION}

A fundamental solution of the inverse Cauchy problem for degenerate parabolic equations of second-order variables with smooth coefficients was constructed first by M. Weber [10]. Under the same conditions on the coefficients, a fundamental solution of the Cauchy problem was constructed in [5], in the case of Holder coefficients for second-order equations with two degenerate groups. The Levy method was modified in [7], and in Banach spaces in [8], for the second order Kolmogorov systems with one degeneracy group [4]. The Kolmogorov equation of high order has features that make it easy to use the Levy method for constructing a fundamental solution. The parametric method was applied to a degenerate parabolic equation of high order with one group of parabolic degeneracy variables in $[2,3,9]$ and with two degenerate groups in [1] and with four degenerate groups for Kolmogorov type systems of the second order in [6]. We modified the Levy method with respect to the properties of a fundamental solution of high-order Kolmogorov-type equations with coefficients dependent only on $t$, in particular a selected point which is a parameter so that an exponential estimate of the fundamental solution and its derivatives is conveniently used.

\section{DEsignATION, TASK STATEMENT AND MAIN RESUlTS}

Let us denote by $n_{j} \in N, j=\overline{1, p}, n_{1} \geq n_{2} \geq \cdots \geq n_{p}, n_{0}=\sum_{j=1}^{p} n_{j}, x=\left(x_{1}, \ldots, x_{p}\right)$, $x_{j}=\left(x_{j 1}, \ldots, x_{j n_{j}}\right), x_{j} \in R^{n_{j}}, x \in R^{n_{0}}, \xi=\left(\xi_{1}, \ldots, \xi_{p}\right), \xi_{j}=\left(\xi_{j 1}, \ldots, \xi_{j n_{j}}\right), \xi_{j} \in R^{n_{j}}, \xi \in R^{n_{0}}$, 
$x^{(j)}=\left(x_{1}, \ldots, x_{j}\right) \in R^{\sum_{k=1}^{j} n_{k}}, \xi^{(j)}=\left(\xi_{1}, \ldots, \xi_{j}\right) \in R^{\sum_{k=1}^{j} n_{k}}, j=\overline{2, p} . \Gamma(\alpha)$ is Euler's gamma function and $B(a, b)$ is Euler's beta function.

$$
\begin{aligned}
x_{j}-\xi_{j}+\sum_{k=1}^{j-1} x_{k} \frac{(t-\tau)^{j-k}}{(j-k) !} & =\left(x_{j 1}-\xi_{j 1}+\sum_{k=1}^{j-1} x_{k 1} \frac{(t-\tau)^{j-k}}{(j-k) !}, . ., x_{j n_{j}}-\xi_{j n_{j}}+\sum_{k=1}^{j-1} x_{k n_{j}} \frac{(t-\tau)^{j-k}}{(j-k) !}\right), \\
\rho_{1}\left(t, x_{1}, \tau, \xi_{1}\right) & =\left(\left|x_{1}-\xi_{1}\right|(t-\tau)^{-\frac{1}{2 b}}\right)^{q}, q=\frac{2 b}{2 b-1}, b \in N, \\
\rho_{j}\left(t, x^{(j)}, \tau, \xi^{(j)}\right) & =\left(\left|x_{j}-\xi_{j}+\sum_{k=1}^{j-1} x_{k} \frac{(t-\tau)^{j-k}}{(j-k) !}\right|(t-\tau)^{-\left(j-1+\frac{1}{2 b}\right)}\right)^{q}, j=\overline{2, p}, \\
\xi(t, \tau) & =\left(\xi_{1}, \xi_{2}-\xi_{1}(t-\tau), \ldots, \xi_{p}+\sum_{k=1}^{p-1}(-1)^{p-k} \xi_{k} \frac{(t-\tau)^{p-k}}{(p-k) !}\right) .
\end{aligned}
$$

We investigate the Cauchy problem for the equation

$$
\partial_{t} u(t, x)-\sum_{j=1}^{p-1} \sum_{\mu=1}^{n_{j+1}} x_{j \mu} \partial_{x_{j+1} \mu} u(t, x)=\sum_{|k| \leq 2 b} a_{k}(t, x) D_{x_{1}}^{k} u(t, x),
$$

with the initial condition

$$
\left.u(t, x)\right|_{t=\tau}=u_{0}(x), \quad 0 \leq \tau \leq t \leq T,
$$

where $\tau$ is a fixed number, and operator

$$
\partial_{t}-\sum_{|k| \leq 2 b} a_{k}(t, x) D_{x_{1}}^{k}, \quad D_{x_{1}}^{k}=\frac{(-1)^{k} \partial^{k_{1}+\cdots+k_{n_{1}}}}{\partial x_{1}^{k_{1}} \ldots \partial x_{n_{1}}^{k_{n_{1}}}}, \quad|k|=k_{1}+\cdots+k_{n_{1}},
$$

is uniformly parabolic in the sense of Petrovsky in the strip $\Pi_{[0, T]}=(t, x), x \in R^{n_{0}}, 0 \leq t \leq T$.

Let us suppose that

1) $a_{k}(t, x), \partial_{x_{j}} a_{k}(t, x), j=\overline{2, p}$, are continuous and bounded in $\Pi_{[0, T]}$,

2) there are constants $\alpha \in(0,1], r \in(0,1]$, such that for any $x \in R^{n_{0}}, \xi \in R^{n_{0}}$ and $t \in[0, T]$

$$
\begin{gathered}
\left|a_{k}(t, x)-a_{k}(t, \xi)\right| \leq c_{1}\left(\left|x_{1}-\xi_{1}\right|^{\alpha}+\sum_{j=2}^{p}\left|x_{j}-\xi_{j}\right|\right), \\
\left|\partial_{x_{j}} a_{k}(t, x)-\partial_{x_{j}} a_{k}(t, \xi)\right| \leq c_{1}|x-\xi|^{r}, \quad j=\overline{2, p} .
\end{gathered}
$$

Theorem 1. If conditions 1)-2) are satisfied, then equation (1) has a fundamental solution of the Cauchy problem (1)-(2) $Z(t, x ; \tau, \xi)$ at $t>\tau$ and the following estimations hold:

$$
\begin{gathered}
\left|\partial_{x_{j}} Z(t, x ; \tau, \xi)\right| \leq A(t-\tau)^{-\sum_{s=1}^{p} \frac{2 b(s-1)+1}{2 b}\left(n_{s}+\left|m_{s}\right|\right)} \Phi(t, x ; \tau, \xi), \\
m_{s}=0, \text { at } s \neq j, m_{j}=1, j=\overline{2, p} ; \\
\left|\partial_{x_{1}}^{m_{1}} Z(t, x ; \tau, \xi)\right| \leq A_{m_{1}}(t-\tau)^{-\frac{n_{1+}\left|m_{1}\right|}{2 b}-\sum_{s=2}^{p} \frac{2 b(s-1)+1}{2 b} n_{s}} \Phi(t, x ; \tau, \xi),
\end{gathered}
$$


$\left|m_{1}\right| \leq 2 b, x \in R^{n_{0}}, \xi \in R^{n_{0}}, 0 \leq \tau<t \leq T$, where

$$
\begin{aligned}
\Phi(t, x ; \tau, \xi)=\sum_{i=1}^{\infty} A^{s} \Gamma & \left(1+\frac{s \alpha^{*}}{2 b}\right) \Gamma\left(\frac{\alpha^{*}}{2 b}\right) \Gamma^{-1}\left(1+\frac{\alpha^{*}(1+s)}{2 b}\right) \\
& \times \exp \left\{-c_{0} \rho_{1}\left(t, x_{1}, \tau, \xi_{1}\right)-2^{-2 s p} c_{0} \sum_{j=2}^{p} \rho_{j}\left(t, x^{(j)}, \tau, \xi^{(j)}\right)\right\},
\end{aligned}
$$

and positive constants $A, A_{m_{1}}, c_{0}$ depend on $n_{0}, 2 b, c_{1}, \alpha, r$, and the constant of parabolicity of the operator (3) is sup $\left|a_{k}(t, x)\right|$ and $\alpha^{*}=\min (\alpha, r)$.

$$
(t, x) \in \Pi_{[0, T]}
$$

Proof. To prove the theorem, we write equation (1) in the form

$$
\begin{aligned}
\partial_{t} u(t, x) & -\sum_{j=1}^{p-1} \sum_{\mu=1}^{n_{j+1}} x_{j \mu} \partial_{x_{j+1} \mu} u(t, x)=\sum_{|k|=2 b} a_{k}(t, \xi(t, \tau)) D_{x_{1}}^{k} u(t, x) \\
& +\sum_{|k|=2 b}\left[a_{k}(t, x)-a_{k}(t, \xi(t, \tau))\right] D_{x_{1}}^{k} u(t, x)+\sum_{|k|<2 b} a_{k}(t, x) D_{x_{1}}^{k} u(t, x) .
\end{aligned}
$$

Let us denote by $Z_{0}(t, x ; \tau, \xi ; \xi(t, \tau))$ the fundamental solution of equation

$$
\partial_{t} u(t, x)-\sum_{j=1}^{p-1} \sum_{\mu=1}^{n_{j+1}} x_{j \mu} \partial_{x_{j+1}} u(t, x)=\sum_{|k|=2 b} a_{k}(t, \xi(t, \tau)) D_{x_{1}}^{k} u(t, x) .
$$

Fundamental solution $Z_{0}(t, x, ; \tau, \xi ; \xi(t, \tau))$ of equation (5) is constructed in [5], where $\xi$ is fixed. For derivatives of $Z_{0}(t, x ; \tau, \xi ; \xi(t, \tau))$ the following inequalities are performed

$$
\begin{aligned}
\left|\partial_{x}^{m} Z_{0}(t, x ; \tau, \xi ; \xi(t, \tau))\right| & \leq C_{m}(t-\tau)^{-\sum_{s=1}^{p} \frac{2 b(s-1)+1}{2 b}\left(n_{s}+\left|m_{s}\right|\right)} \\
& \times \exp \left\{-c_{0}\left(\sum_{j=2}^{p} \rho_{j}\left(t, x^{(j)}, \tau, \xi^{(j)}\right)+\rho_{1}\left(t, x_{1}, \tau, \xi_{1}\right)\right)\right\}
\end{aligned}
$$

where $|m|=\sum_{j=1}^{p}\left|m_{j}\right|,\left|m_{j}\right|=\sum_{k=1}^{n_{j}} m_{j k}, t>\tau, C_{m}>0$.

Fundamental solution $Z(t, x ; \tau, \xi)$ of equation (1) will be sought in the form

$$
Z(t, x ; \tau, \xi)=Z_{0}(t, x ; \tau, \xi ; \xi(t, \tau))+\int_{\tau}^{t} d \beta \int_{R^{n_{0}}} Z_{0}(t, x ; \beta, \gamma ; \gamma(t, \beta)) \varphi(\beta, \gamma ; \tau, \xi) d \gamma,
$$

where $\varphi(t, x ; \tau, \xi)$ is an unknown absolutely integrable on $R^{n_{0}}$ function at $t>\tau$.

We substitute (6) into equation (1) with respect to the function $\varphi(t, x ; \tau, \xi)$, then

$$
\varphi(t, x ; \tau, \xi)=K(t, x ; \tau, \xi)+\int_{\tau}^{t} K(t, x ; \beta, \gamma) \varphi(\beta, \gamma ; \tau, \xi) d \gamma
$$

where

$$
\begin{aligned}
K(t, x ; \tau, \xi) & =\sum_{|k|=2}\left(a_{k}(t, x)-a_{k}(t, \xi(t, \tau))\right) D_{x_{1}}^{k} Z_{0}(t, x ; \tau, \xi ; \xi(t, \tau)) \\
& +\sum_{|k|<2 b} a_{k}(t, x) D_{x_{1}}^{k} Z_{0}(t, x ; \tau, \xi ; \xi(t, \tau)) .
\end{aligned}
$$


The solution of equation (8) can be represented by a Neumann series

$$
\varphi(t, x ; \tau, \xi)=\sum_{n=1}^{\infty} K_{n}(t, x ; \tau, \xi)
$$

where

$$
K(t, x ; \tau, \xi)=K_{1}(t, x ; \tau, \xi) ; \quad K_{n}(t, x ; \tau, \xi)=\int_{\tau}^{t} d \beta \int_{R^{n_{0}}} K(t, x ; \beta, \gamma) K_{n-1}(\beta, \gamma ; \tau, \xi) d \gamma
$$

Let us show the convergence of series (9) and the required estimation of the function for the Levy method $\varphi(t, x ; \tau, \xi)$ and its increments.

Using the following lemma, which generalizes Lemma 2 and Lemma 1 in [3] for equation (1), we can obtain estimates for $K_{n}(t, x ; \tau, \xi)$ and $K(t, x ; \tau, \xi)$.

Lemma 1. For any points $(t, x),(\beta, \xi),(\tau, y), 0 \leq \tau<\beta<t, x \in R^{n_{0}}, \xi \in R^{n_{0}}, y \in R^{n_{0}}$, $b \in N, 2 b>2$ the following inequality holds

$$
\begin{aligned}
\rho_{1}\left(t, x_{1}, \beta, \xi_{1}\right) & +\sum_{j=2}^{p} \rho_{j}\left(t, x^{(j)}, \beta, \xi^{(j)}\right)+\rho_{1}\left(\beta, \xi_{1}, \tau, y_{1}\right)+\sum_{j=2}^{p} \rho_{j}\left(\beta, \xi^{(j)}, \tau, y^{(j)}\right) \\
& \geq 2^{-2 p}\left(\sum_{j=2}^{p} \rho_{j}\left(t, x^{(j)}, \tau, y^{(j)}\right)+\rho_{1}\left(t, x_{1}, \tau, y_{1}\right)\right) .
\end{aligned}
$$

The proof of Lemma 1 is based on the inequalities

$$
\begin{aligned}
& \rho_{p}\left(t, x^{(p)}, \beta, \xi^{(p)}\right)+\rho_{p}\left(\beta, \xi^{(p)}, \tau, y^{(p)}\right) \\
& \quad \geq 2^{-2}\left(\left|x_{p}-y_{p}+\sum_{j=1}^{p-1}\left[x_{k}(t-\beta)^{p-k}+\xi_{k}(\beta-\tau)^{p-k}\right] \frac{1}{(p-k) !}\right|(t-\tau)^{-p+1-\frac{1}{2 b}}\right)^{q} .
\end{aligned}
$$

From (12) we can get

$$
\begin{gathered}
\left(\left|x_{p}-y_{p}+\sum_{k=1}^{p-1}\left[x_{k}(t-\beta)^{p-k}+\xi_{k}(\beta-\tau)^{p-k}\right]((p-k) !)^{-1}\right|(t-\tau)^{-p+1-\frac{1}{2 b}}\right)^{q} \\
\geq 2^{-2}\left(\mid x_{p}-y_{p}+\sum_{k=1}^{p-1}\left[x_{k}(t-\beta)^{p-k}+\xi_{k}(\beta-\tau)^{p-k}\right]((p-k) !)^{-1}\right. \\
\left.\quad \times \frac{x_{1}\left((\beta-\tau)^{p-1}+(t-\beta)^{p-1}\right)}{(p-1) !} \mid(t-\tau)^{-p+1-\frac{1}{2 b}}\right)^{q} \\
-\sum_{\mu=1}^{n_{p}}\left(\left[\left|x_{1 \mu}-\xi_{1 \mu}\right|(\beta-\tau)^{p-1}\right]((p-k) !)^{-1}(t-\tau)^{-p+1-\frac{1}{2 b}}\right)^{q} .
\end{gathered}
$$

Applying (12) to the first part of (13) $(p-2)$ times, we have

$$
\left(\left|x_{p}-y_{p}+\sum_{k=1}^{p-1}\left(x_{k}(t-\beta)^{p-k}+\xi_{k}(\beta-\tau)^{p-k}\right)((p-k) !)^{-1}\right|(t-\tau)^{-p+1-\frac{1}{2 b}}\right)^{q}
$$




$$
\begin{aligned}
& \left.\geq 2^{-2(p-1)} \rho_{p}\left(t, x^{(p)}, \tau, y^{(p)}\right)-\sum_{\mu=1}^{n_{p}}\left(\left|x_{j \mu}-\xi_{j \mu}\right|(\beta-\tau)^{p-1}\right)((p-1) !)^{-1}(t-\tau)^{-p+1-\frac{1}{2 b}}\right)^{q} \\
& -\sum_{j=2}^{p-1} \sum_{\mu=2}^{n_{j}} 2^{-2(j-1)}\left(\left|x_{1 \mu}-\xi_{1 \mu}+\sum_{k=2}^{j-1} x_{k \mu}(t-\beta)^{j-k}((j-k) !)^{-1}\right| \frac{(\beta-\tau)^{p-j}}{(p-j) !}(t-\tau)^{-p+1-\frac{1}{2 b}}\right)^{q} .
\end{aligned}
$$

Taking into account the inequalities (11)-(14), we get

$$
\begin{aligned}
& \rho_{p}\left(t, x^{(p)}, \beta, \xi^{(p)}\right)+\rho_{p}\left(\beta, \xi^{(p)}, \tau, y^{(p)}\right) \geq 2^{-2 p} \rho_{p}\left(t, x^{(p)}, \tau, y^{(p)}\right) \\
& -\sum_{j=2}^{p-1} \sum_{\mu=1}^{n_{p}} 2^{-2(j-1)}\left(\left|x_{j \mu}-\xi_{j \mu}+\sum_{k=2}^{j-1} x_{k \mu}(t-\beta)^{j-k}((j-k) !)^{-1}\right|(\beta-\tau)^{p-j}\left((p-j)^{!}\right)^{-1}\right. \\
& \left.\times(t-\tau)^{-p+j-\frac{1}{2 b}}\right)^{q}-2^{-2} \sum_{\mu=1}^{n_{p}}\left(\left|x_{1 \mu}-\xi_{1 \mu}\right|(\beta-\tau)^{p-1}((p-1) !)^{-1}(t-\tau)^{-p+1-\frac{1}{2 b}}\right)^{q} .
\end{aligned}
$$

We will collect all of the terms that contain $x_{p-1}-\xi_{p-1}$

$$
\begin{aligned}
& \rho_{p-1}\left(\beta, x^{(p-1)}, \beta, \xi^{(p-1)}\right)-2^{-2(p-1)} \sum_{\mu=1}^{n_{p}}\left(\mid x_{p-1 \mu}-\xi_{p-1 \mu}+\sum_{k=1}^{p-2} x_{k}(t-\beta)^{p-1-k}\right. \\
& \left.\quad \times \frac{1}{((p-1-k) !)} \mid(\beta-\tau)(t-\beta)^{-p+1-\frac{1}{2 b}}\right)^{q} \geq \sum_{\mu=n_{p+1}}^{n_{p-1}}\left(\mid x_{p-1 \mu}-\xi_{p-1 \mu}\right. \\
& \left.+\sum_{k=1}^{p-2} x_{k \mu} \frac{(t-\beta){ }^{p-1-k}}{(p-1-k) !} \mid(t-\beta)^{-p+3-\frac{1}{2 b}}\right)^{q}+\sum_{\mu=1}^{n_{p}}\left(1-2^{-2(p-1)}\right) \\
& \quad \times\left(\left|x_{p-1 \mu}-\xi_{p-1 \mu}+\sum_{k=1}^{p-2} x_{k \mu}(t-\beta)^{p-1-k}((p-1-k) !)^{-1}\right|(t-\tau)^{-p+2-\frac{1}{2 b}}\right)^{q} .
\end{aligned}
$$

Repeating all inequalities (12), (16) for the terms $\rho_{j}\left(t, x^{(j)}, \beta, \xi^{(j)}\right)+\rho_{j}\left(\beta, \xi^{(j)}, \tau, y^{(j)}\right)$, $j=\overline{1, p-1}$, and adding their together we have

$$
\begin{aligned}
\rho_{1}\left(t, x_{1}, \beta, \xi_{1}\right) & +\rho_{1}\left(\beta, \xi_{1}, \tau, y_{1}\right)+\sum_{j=2}^{p}\left(\rho_{j}\left(t, x^{(j)}, \beta, \xi^{(j)}\right)+\rho_{j}\left(\beta, \xi^{(j)}, \tau, y^{(j)}\right)\right) \\
& \geq 2^{-2 p}\left(\sum_{j=2}^{p} \rho_{j}\left(t, x^{(j)}, \tau, y^{(j)}\right)+\rho_{1}\left(t, x_{1}, \tau, y_{1}\right)\right) .
\end{aligned}
$$

Lemma 2. The following estimations are performed for reproducing kernels:

$$
\begin{aligned}
\left|K_{m}(t, x ; \tau, \xi)\right| & \leq A_{m}^{m}(t-\tau)^{-\sum_{j=1}^{p} \frac{(1+2 b(j-1)) n_{j}}{2 b}-1+\frac{m \alpha}{2 b}} \\
& \times \exp \left\{\rho_{1}\left(t, x_{1}, \beta, \xi_{1}\right)-2^{-2 p m} c \sum_{j=2}^{p} \rho_{j}\left(t, x^{(j)}, \tau, \xi^{(j)}\right)\right\},
\end{aligned}
$$

at $m \leq m^{*}=\left[\sum_{j=1}^{p} \frac{\left((1+2 b(j-1)) n_{j}+2 b\right)}{\alpha}\right]+1$; 


$$
\begin{aligned}
\left|K_{m+l}(t, x ; \tau, \xi)\right| & \leq A_{m}^{m+l} \prod_{k=0}^{l-1} B\left(\frac{\alpha}{2 b}, 1+\frac{\alpha k}{2 b}\right)(t-\tau)^{\frac{\alpha l}{2 b}} \\
& \times \exp \left\{-c \rho_{1}\left(t, x_{1}, \beta, \xi_{1}\right)-2^{-2 p(m+l)} \sum_{j=2}^{p} \rho_{j}\left(t, x^{(j)}, \tau, \xi^{(j)}\right)\right\},
\end{aligned}
$$

at $m+l>m^{*}$.

From (17), (18) it follows the convergence of a series (9) following for $\varphi(t, x ; \tau, \xi)$

$$
|\varphi(t, x ; \tau, \xi)| \leq A(t-\tau)^{-\sum_{j=1}^{p} \frac{(1+2 b(j-1)) n_{j}+2 b-\alpha}{2 b}} \Phi(t, x ; \tau, \xi) .
$$

Let us prove the existence of derivatives $\partial_{x_{j}} \varphi(t, x ; \tau, \xi), j=\overline{2, p}$, at $t>\tau$.

Under the assumption 1), there are continuous derivatives $\partial_{x_{j}} K(t, x ; \tau, \xi), j=\overline{2, p}$ satisfying the estimations

$$
\begin{aligned}
\left|\partial_{x_{j}} K(t, x ; \tau, \xi)\right| & \leq A \exp \left\{-c\left(\sum_{j=2}^{p} \rho_{j}\left(t, x^{(j)}, \tau, \xi^{(j)}\right)+\rho_{1}\left(t, x_{1}, \tau, \xi_{1}\right)\right)\right\} \\
& \times(t-\tau)^{-\sum_{s=1}^{p} \frac{(2 b(s-1)+1) n_{s}}{2 b}-\frac{j-\left(1-\alpha^{*}\right)}{2 b}}, \quad t>\tau .
\end{aligned}
$$

To prove the existence of derivatives $\partial_{x_{j}} K(t, x ; \tau, \xi), j=\overline{2, p}$, we use the following property of the fundamental solution of equation (5) with $\xi(t, \tau)=y$, where $y$ is a parameter

$$
\partial_{t} u(t, x)-\sum_{j=1}^{p-1} \sum_{\mu=1}^{n_{j+1}} x_{j \mu} \partial_{x_{j+1} \mu} u(t, x)=\sum_{|k| \leq 2 b} a_{k}(t, \xi(t, \tau)) D_{x_{1}}^{k} u(t, x) .
$$

Property 1. If $a_{k}(t, y)$ have continuous bounded derivatives by the parameter $y$ up to the order $r$, then there are continuous derivatives by $y, \partial_{y}^{s} \partial_{x_{1}}^{m} Z_{0}(t, x ; \tau, \xi ; y), s \in \overline{0, r}$, and

$$
\begin{aligned}
\left|\partial_{x_{1}}^{m} \partial_{y}^{s} Z_{0}(t, x ; \tau, \xi ; y)\right| & \leq C_{m} \exp \left\{-c\left(\sum_{j=2}^{p} \rho_{j}\left(t, x^{(j)}, \tau, \xi^{(j)}\right)+\rho_{1}\left(t, x_{1}, \tau, \xi_{1}\right)\right)\right\} \\
& \times(t-\tau)^{-\sum_{j=1}^{p} \frac{\left(1+2 b(j-1) n_{j}\right.}{2 b}-\frac{|m|}{2 b}} .
\end{aligned}
$$

Let us consider $\partial_{x_{p \mu}} K(t, x ; \beta, \gamma), \mu=\overline{1, n_{p}}$. Then

$$
\begin{aligned}
& \partial_{x_{p \mu}} K(t, x ; \beta, \gamma)=\sum_{|k|=2 b}\left(\partial_{x_{p \mu}} a_{k}(t, x)-\partial_{\gamma_{p \mu}} a_{k}(t, \gamma(t, \beta))\right) \\
& \quad \times D_{x_{1}}^{k} Z_{0}(t, x ; \beta, \gamma ; \gamma(t, \beta))+\sum_{|k|=2 b}\left(\partial_{\gamma_{p \mu}} a_{k}(t, \gamma(t, \beta))\right) D_{x_{1}}^{k} Z_{0}(t, x ; \beta, \gamma ; \gamma(t, \beta)) \\
& +\sum_{|k|=2 b}\left(a_{k}(t, x)-a_{k}(t, \gamma(t, \beta))\right) \partial_{x_{p \mu}} D_{x_{1}}^{k} Z_{0}(t, x ; \beta, \gamma ; \gamma(t, \beta)) \\
& +\sum_{|k|<2 b} \partial_{x_{p \mu}} a_{k}(t, x) D_{x_{1}}^{k} Z_{0}(t, x ; \beta, \gamma ; \gamma(t, \beta)) .
\end{aligned}
$$


Let us rewrite (22) by a convenient form for applications

$$
\begin{aligned}
& \partial_{x_{p \mu}} K(t, x ; \beta, \gamma)=\sum_{|k|=2 b}\left(\partial_{x_{p \mu}} a_{k}(t, x)-\partial_{\gamma_{p \mu}} a_{k}(t, \gamma(t, \beta))\right) \\
& \quad \times D_{x_{1}}^{k} Z_{0}(t, x ; \beta, \gamma ; \gamma(t, \beta))-\partial_{\gamma_{p \mu}}\left(\sum_{|k|=2 b}\left(a_{k}(t, x)-a_{k}(t, \gamma(t, \beta))\right)\right. \\
& \quad \times D_{x_{1}}^{k} Z_{0}(t, x ; \beta, \gamma ; \gamma(t, \beta))+\sum_{|k|=2 b}\left(a_{k}(t, x)-a_{k}(t, \gamma(t, \beta))\right. \\
& \quad \times\left. D_{x_{1}}^{k} \partial_{\bar{\gamma}_{p \mu}} D_{x_{1}}^{k} Z_{0}(t, x ; \beta, \gamma ; \bar{\gamma}(t, \beta))\right|_{\bar{\gamma}=\gamma}+\sum_{|k|<2 b}\left(a_{k}(t, x)\right)_{x_{p \mu}}^{\prime} D_{x_{1}}^{k} Z_{0}(t, x ; \beta, \gamma ; \gamma(t, \beta)) \\
& +\left.\sum_{|k|<2 b} a_{k} \partial_{\bar{\gamma}_{p \mu}} D_{x_{1}}^{k} Z_{0}(t, x ; \beta, \gamma ; \bar{\gamma}(t, \beta))\right|_{\bar{\gamma}=\gamma}-\sum_{|k|<2 b} a_{k}(t, x) D_{x_{1}}^{k} \partial_{\gamma_{p \mu}} Z_{0}(t, x ; \beta, \gamma ; \gamma(t, \beta)),
\end{aligned}
$$

where $\mu=\overline{1, n_{p}}, \bar{\gamma}=\left(\gamma_{1}, \ldots, \gamma_{p-1}, \overline{\gamma_{p}}\right)$. Using the images (23), estimates (6) and (21) and integrating by parts of the terms with $\partial_{\gamma_{p \mu}}$, we can get $\partial_{x_{p \mu}} K_{2}(t, x ; \tau, \xi)=\lim _{h \rightarrow 0} \int_{0}^{t-h} d \beta \int_{R^{n_{0}}} \partial_{x_{p \mu}}$ $K(t, x ; \beta, \gamma) K(\beta, \gamma ; \tau, \xi) d \gamma$.

From the estimations of reproducing kernel (18), estimations of derivatives of the kernel (20) and Lemma 1, we obtain $\left|\partial_{x_{p \mu}} K_{2}(t, x ; \tau, \xi)\right| \leq A_{2} \exp \left\{-c_{2}(1-\varepsilon)\left(\rho_{1}\left(t, x_{1}, \tau, \xi_{1}\right)\right.\right.$ $\left.\left.+2^{-2 p} \sum_{j=2}^{p} \rho_{j}\left(t, x^{(j)}, \tau, \xi^{(j)}\right)+\rho_{1}\left(t, x_{1}, \tau, \xi_{1}\right)\right)\right\}(t-\tau)^{-\sum_{j=1}^{p} \frac{(1+2 b(j-1)) n_{j}}{2 b}-p-\left(1-\alpha^{*}\right) / 2 b}$ at $t>\tau$. By the method of mathematical induction we can prove the existence $\partial_{x_{p \mu}} K_{m}(t, x ; \tau, \xi)$ for any $m$ and evaluation

$$
\begin{aligned}
\left|\partial_{x_{p \mu}} K_{m}(t, x ; \tau, \xi)\right| & \leq A_{m}(\varepsilon) \exp \left\{-c_{2}(1-\varepsilon m)\left(\rho_{1}\left(t, x_{1}, \tau, \xi_{1}\right)+2^{-m p} \sum_{j=2}^{p} \rho_{j}\left(t, x^{(j)}, \tau, \xi^{(j)}\right)\right.\right. \\
& \left.+\rho_{1}\left(t, x_{1}, \tau, \xi_{1}\right)\right\}(t-\tau)^{-\sum_{j=1}^{p} \frac{(1+2 b(j-1)) n_{j}}{2 b}-p-(1-\alpha m) / 2 b}, \quad \mu=\overline{1, n_{p} .}
\end{aligned}
$$

Taking into account the estimation (24), we can estimate the series $\sum_{m=1}^{\infty} \partial_{x_{p \mu}} K_{m}(t, x ; \tau, \xi)$ by a converging series:

$$
\begin{aligned}
& \left|\sum_{m=1}^{\infty} \partial_{x_{p \mu}} K_{m}(t, x ; \tau, \xi)\right| \leq \sum_{m=1}^{l} A_{m}(t-\tau)-\sum_{j=1}^{p} \frac{\left(1+2 b(j-1) n_{j}\right.}{2 b}-p-\left(1-\alpha^{*} m\right) / 2 b \\
& \times \exp \left\{-c_{2}(1-m \varepsilon)\left(c_{m} \rho_{1}\left(t, x_{1}, \tau, \xi_{1}\right)+2^{-2 m p}\left(\rho_{1}\left(t, x_{1}, \tau, \xi_{1}\right)+\sum_{j=2}^{p} \rho_{j}\left(t, x^{(j)}, \tau, \xi^{(j)}\right)\right)\right\}\right. \\
& \quad+\sum_{k=1}^{\infty} A_{0}\left(\Gamma\left(\frac{\alpha^{*}}{2 b}\right) F A_{0}\right)^{k}(t-\tau)^{\frac{\alpha^{*} k}{2 b}} \Gamma^{-1}\left(1+\frac{k \alpha^{*}}{2 b}\right) \\
& \quad \times \exp \left\{-c_{5}\left(c_{4 l+k+1} \rho_{1}\left(t, x_{1}, \tau, \xi_{1}\right)+2^{-2 p(l+k-1)}\left(\rho_{1}\left(t, x_{1}, \tau, \xi_{1}\right)+\sum_{j=2}^{p} \rho_{j}\left(t, x^{(j)}, \tau, \xi^{(j)}\right)\right)\right\},\right.
\end{aligned}
$$

where $l=\left[\sum_{j=1}^{p} \frac{(1+2 b(j-1)) n_{j}+2 b p+1}{\alpha^{*}}\right]+1$, and $A_{0}, F$ are positive constants,

$$
F=\left(2 \int_{0}^{\infty} \exp \left\{-\frac{\alpha^{2}}{2}\right\} d \alpha\right)^{n_{0}}
$$


The series $\sum_{m=1}^{\infty} \partial_{x_{p}} K_{m}(t, x ; \tau, \xi)$ at $0<\delta \leq t-\tau \leq T$ is convergent uniformly and absolutely. Then $\partial_{x_{p}} \varphi(t, x ; \tau, \xi)=\sum_{m=1}^{\infty} \partial_{x_{p}} K(t, x ; \tau, \xi)$ and $\partial_{x_{p}} K_{m}(t, x ; \tau, \xi)$ are continuous, then in the domain of convergence and $\partial_{x_{p}} \varphi(t, x ; \tau, \xi)$ continuous function. Inequality (25) will be written in the form

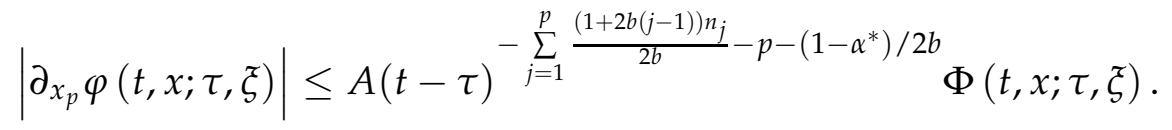

Let us consider $\partial_{x_{j \mu}} K(t, x ; \beta, \gamma), j=\overline{2, p-1}, \mu=\overline{1, n_{j}}$. For $\mu=\overline{n_{j-1}+1, n_{j}}$ formula (23) is true with the corresponding replacing $p$ by $j$. For $\mu=\overline{1, n_{j-1}}, \partial_{x_{j \mu}} K(t, x ; \beta, \gamma)$ can be written in the form

$$
\begin{aligned}
& \partial_{x_{j \mu}} K(t, x ; \beta, \gamma)=\sum_{|k|=2 b}\left[\partial_{x_{j \mu}} a_{k}(t, x)-\left.\partial_{y_{j \mu}} a_{k}(t, y)\right|_{y=\gamma(t, \beta)}\right] D_{x_{1}}^{k} Z_{0}(t, x ; \beta, \gamma ; \gamma(t, \beta)) \\
& -\partial_{\gamma_{j \mu}}\left(\sum_{|k|=2 b}\left[a_{k}(t, x)-a_{k}(t, \gamma(t, \beta))\right] D_{x_{1}}^{k} Z_{0}(t, x ; \beta, \gamma ; \gamma(t, \beta))\right) \\
& +\left.\sum_{|k|=2 b}\left[a_{k}(t, x)-a_{k}(t, \gamma(t, \beta))\right] \partial_{\bar{\gamma}_{j \mu}} D_{x_{1}}^{k} Z_{0}(t, x ; \beta, \gamma ; \bar{\gamma}(t, \beta))\right|_{\gamma}=\gamma \\
& +\left.\sum_{l=1}^{p-j} \sum_{|k|=2 b}\left[a_{k}(t, x)-a_{k}(t, \gamma(t, \beta))\right] \partial_{\gamma_{j+l, \mu}} D_{x_{1}}^{k} Z_{0}(t, x ; \beta, \gamma ; \bar{\gamma}(t, \beta))\right|_{\gamma}=\gamma \\
& \times\left.(-1)^{l} \frac{(t-\tau)^{p-l-l}}{(p-j-l) !} \sum_{|k|=2 b} \partial_{y_{j+l} \mu} a_{k}(t, y)\right|_{y=\gamma(t, \beta)}(-1)^{l} \frac{(t-\beta)^{p-j-l}}{(p-j-l) !} \\
& \times D_{x_{1}}^{k} \partial_{\gamma_{j \mu}} Z_{0}(t, x ; \beta, \gamma ; \gamma(t, \beta))+\sum_{|k|<2 b}\left(a_{k}(t, x)\right)_{x_{j \mu}}^{\prime} D_{x_{1}}^{k} Z_{0}(t, x ; \beta, \gamma ; \gamma(t, \beta)) \\
& +\sum_{|k|<2 b} \partial_{\gamma_{j \mu}} a_{k}(t, x) D_{x_{1}}^{k} Z_{0}(t, x ; \beta, \gamma ; \gamma(t, \beta)) \\
& +\left.\sum_{|k|<2 b} a_{k}(t, x) D_{x_{1}}^{k} \partial_{\bar{\gamma}_{j \mu}} Z_{0}(t, x ; \beta, \gamma ; \bar{\gamma}(t, \beta))\right|_{\bar{\gamma}=\gamma} \\
& +(-1)^{l} \frac{(t-\beta)^{p-j-l}}{(p-j-l) !} \sum_{l=1}^{p-j} \sum_{|k|=2 b} a_{k}(t, x) D_{x_{1}}^{k} \partial_{\gamma_{j+l}} Z_{0}(t, x ; \beta, \gamma ; \gamma(t, \beta)) .
\end{aligned}
$$

Kernels have the highest singularity at the variable $x_{p}$. Also, using (26) we have the existence of $\partial_{x_{j}} \varphi(t, x ; \tau, \xi), j=\overline{2, p-1}$ and the following estimations

$$
\left|\partial_{x_{j}} \varphi(t, x ; \tau, \xi)\right| \leq A(t-\tau)^{-\sum_{s=1}^{p} \frac{(1+2 b(j-1)) n_{s}-\alpha^{*}+1}{2 b}-j} \Phi(t, x ; \tau, \xi), \quad j=\overline{2, p-1} .
$$

Using arguments like in [1] we can get

$$
\Delta_{h x_{1}} \varphi(t, x ; \tau, \xi)=\Delta_{h x_{1}} K(t, x ; \tau, \xi)+\int_{\tau}^{t} d \beta \int_{R^{n_{0}}} \Delta_{h x_{1}} K(t, x ; \beta, \gamma) K(\beta, \gamma ; \tau, \xi) d \gamma
$$


Applying the technique developed for parabolic systems in [6], and the evaluation of reproducing kernels, we obtain

$$
\begin{gathered}
\left|\Delta_{h x_{1}} \varphi(t, x ; \tau, \xi)\right| \leq\left|h_{x_{1}}\right|^{\alpha_{1}}(t-\tau)^{-\sum_{s=1}^{p} \frac{(1+2 b(s-1)) n_{s}-\left(1-\alpha_{2}\right)}{2 b}-j} \Phi(t, x ; \tau, \xi), \\
\alpha_{1}>0, \alpha_{2}>0, \alpha_{1}+\alpha_{1}=\alpha .
\end{gathered}
$$

The existence and evaluation of $\partial_{x_{1}}^{k} Z(t, x ; \tau, \xi),|k| \leq 2 b$, at $t>\tau$, are established for both of the cases of parabolic equations and systems in [6]. The theorem is proved.

\section{REFERENCES}

[1] Burtnyak I.V., Malytska A. The evaluation of derivatives of double barrier options of the Bessel processes by methods of spectral analysis. Investment Management and Financial Innovations 2017, 14 (3), 126-134. doi:10.21511/imfi.14(3).2017.12

[2] Burtnyak I.V., Malytska A. Spectral study of options based on CEV model with multidimensional volatility. Investment Management and Financial Innovations 2018, 15 (1), 18-25. doi:10.21511/imfi.15(1).2018.03

[3] Burtnyak I.V., Malytska A. Taylor expansion for derivative securities pricing as a precondition for strategic market decisions. Problems and Perspectives in Management 2018, 16 (1), 224-231. doi:10.21511/ppm.16(1).2018.22

[4] Burtnyak I.V., Malyts'ka H.P. On the Fundamental Solution of the Cauchy Problem for Kolmogorov Systems of the Second Order. Ukr. Math. J. 2019, 70 (1), 1275-1287. doi:10.1007/s11253-018-1568-y

[5] Cinti C., Pascucci A. Polidoro S. Pointwise estimates for a class of nonhomogeneous Kolmogorov equations. Math. Ann. 2008, 340 (2), 237-264. doi:10.1007/s00208-007-0147-6

[6] Eidelman S.D. Parabolic systems. North-Holland, Amsterdam, 1969.

[7] Eidelman S.D., Ivasyshen S.D., Malytska H.P. A modified Levi method: development and application. Dopov. Nats. Akad. Nauk Ukr. Mat. Prirodozn. Tekh. Nauki. 1998, 5 (2), 14-19.

[8] Ivasyshen S.D., Medyns'kyi I.P. On the Classical Fundamental Solutions of the Cauchy Problem for Ultraparabolic Kolmogorov-Type Equations with Two Groups of Spatial Variables. J. Math. Sci. (N.Y.) 2018, 4 (231), 507-526. doi:10.1007/s10958-018-3830-0

[9] Malitskaya A. P. Construction of the fundamental solutions of certain higher-order ultraparabolic equations. Ukr. Mat. Zh. 1985, 37 (6), 713-718.

[10] Weber M. The Fundamental Solution of a Degenerate Partial Differential Equation of Parabolic Type. Trans. Amer. Math. Soc. 1951, 71 (1), 24-37. doi:10.2307/1990857

Received 08.05.2019

Малицька Г.П., Буртняк І.В. Побудова фундаментального розв' язку одного класу вироджених параболічних рівнянь високого порядку // Карпатські матем. публ. - 2020. - Т.12, №1. - С. $79-87$.

У статті модифікованим методом Аеві побудовано функцію Гріна для одного класу ультрапараболічних рівнянь високого порядку з довільною кількістю груп виродження параболічності. Модифікований метод Аеві розроблено для рівнянь Колмогорова високого порядку з коефіцієнтами залежними від усіх змінних, при цьому заморожена точка, яка є параметриксом, підібрана так, щоб зручно використовувалася експоненціальна оцінка фундаментального розв' язку та його похідних.

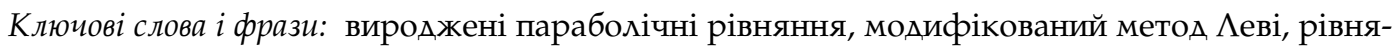
ння Колмогорова, фундаментальний розв'язок, параметрикс. 